\title{
Bond Character of Carbon Cones and Discs
}

\author{
F.S. Hage, ${ }^{*}, * * \varnothing$. Prytz, ${ }^{* *}$ R. Brydson, ${ }^{* * *}$ A.E. Gunnæs, ${ }^{* *}$ and G. Helgesen, ${ }^{*}, * *$ \\ * Physics Department, Institute for Energy Technology, NO-2027 Kjeller, Norway, \\ ** Department of Physics, University of Oslo, NO-0316 Oslo, Norway, \\ *** Institute for Materials Research, University of Leeds, Leeds LS2 9JT, U.K.
}

Carbon cones have been named "the fifth form of carbon" and their unique topography make them promising for applications such as hydrogen storage, sensors and electrodes [1]. Cones and discs were produced by pyrolysis of heavy oil with a plasma arc in the Kvaerner Carbon Black and Hydrogen Process [2]. A subsequent study by Krishnan et al. reported that the observed cone apex angles of $112.9^{\circ}, 84.6^{\circ}, 60^{\circ}, 38.9^{\circ}$ and $19.2^{\circ}$ corresponded to the incorporation of $1-560^{\circ}$ disclinations in a hexagonal graphitic sheet [3]. The discs may be regarded as cones with apex angles of $180^{\circ}$. A bright field micrograph of two cones supported by a holey carbon film is shown in figure 1.

It is of great interest to determine the electronic structure of these cones and discs for future applications. Electron Energy Loss Spectroscopy (EELS) in the TEM is an excellent technique for studying the electronic structure of each individual particle. In this work we present an investigation of the bond character of cones and discs by analysis of the carbon $\mathrm{K}$ ionization edge for a range of samples heat treated up to $2700^{\circ} \mathrm{C}$. Sample orientation is known to affect the edge intensities due the anisotropy on graphitic structures. However, for a certain magic collection semi- angle, the normal and perpendicular scattering components are weighted so edge intensities are independent of sample orientation [4]. This collection condition was employed for accurate determination of edge intensities.

Bonding information was extracted by the method described by Zhili et al. [5]. Three Gaussians were fitted to the experimental ionization edges corresponding to a $\mathrm{C}=\mathrm{C} \pi^{*}$ component (ca. $285 \mathrm{eV}$ ), $\mathrm{C}-\mathrm{C} \sigma^{*}$ component (ca. $292 \mathrm{eV}$ ) and $\mathrm{C}=\mathrm{C} \sigma^{*}$ component (ca. $300 \mathrm{eV}$ ). The $\mathrm{sp}^{2}$ bonding character was then determined from the $\pi^{*}$ component normalized to spectra from $100 \% \mathrm{sp}^{2}$ hybridized graphite (highly ordered pyrolytic graphite) [5]. Figure 2 shows a fitted carbon K ionization edge of a disc heat treated at $1600^{\circ} \mathrm{C}$. A residual peak (ca. $287 \mathrm{eV}$ ) can be seen between the $\pi^{*}$ and $\sigma^{*}$ fits in figure 2. Zhili at al. suggested that this feature can be used as a measure of fullerene character [5]. We discuss this for the case of carbon cones and discs [6].

\section{References}

[1] J. Muller et al., Y. Magarshak (ed.), Silicon Versus Carbon, Springer Netherlands, 2009 p285

[2] J.A. Bakken et al., Pure and Applied Chemistry, 70 (1998) 1223

[3] A. Krishnan et al., Nature, 388 (1997) 451

[4] H.R Daniels et al., Ultramicroscopy, 96 (2003) 523

[5] Z. Zhili et al., Journal of Physics: Conference Series, 241 (2010) 012094

[6] This work was supported by the Research Council of Norway under Contract No. 191621/V30 


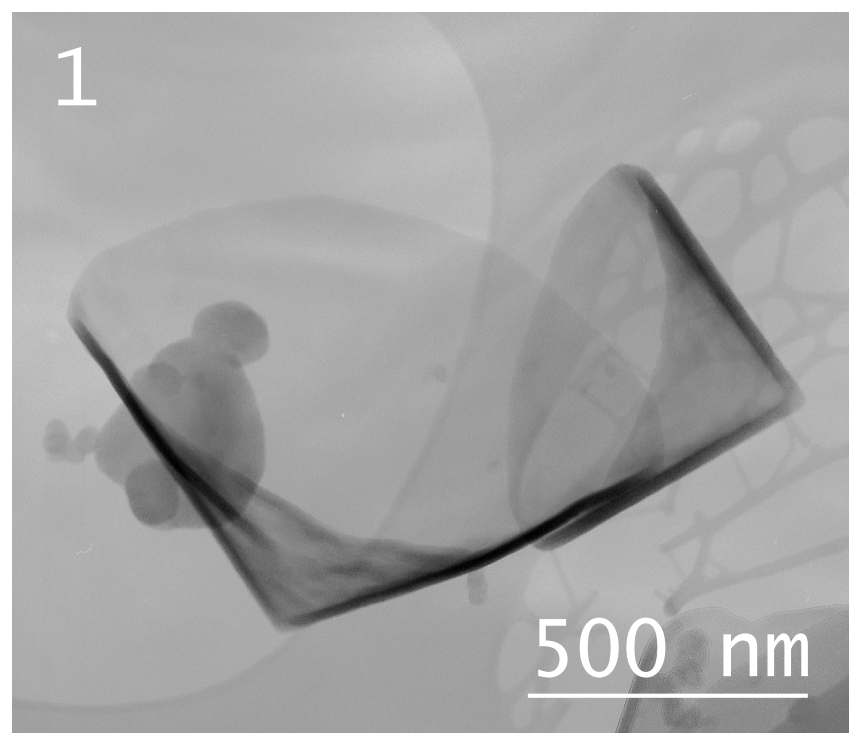

FIG. 1. A bright field micrograph of two non- heat treated cones. The particle attached to the largest cone is a cluster of carbon onion like structures.

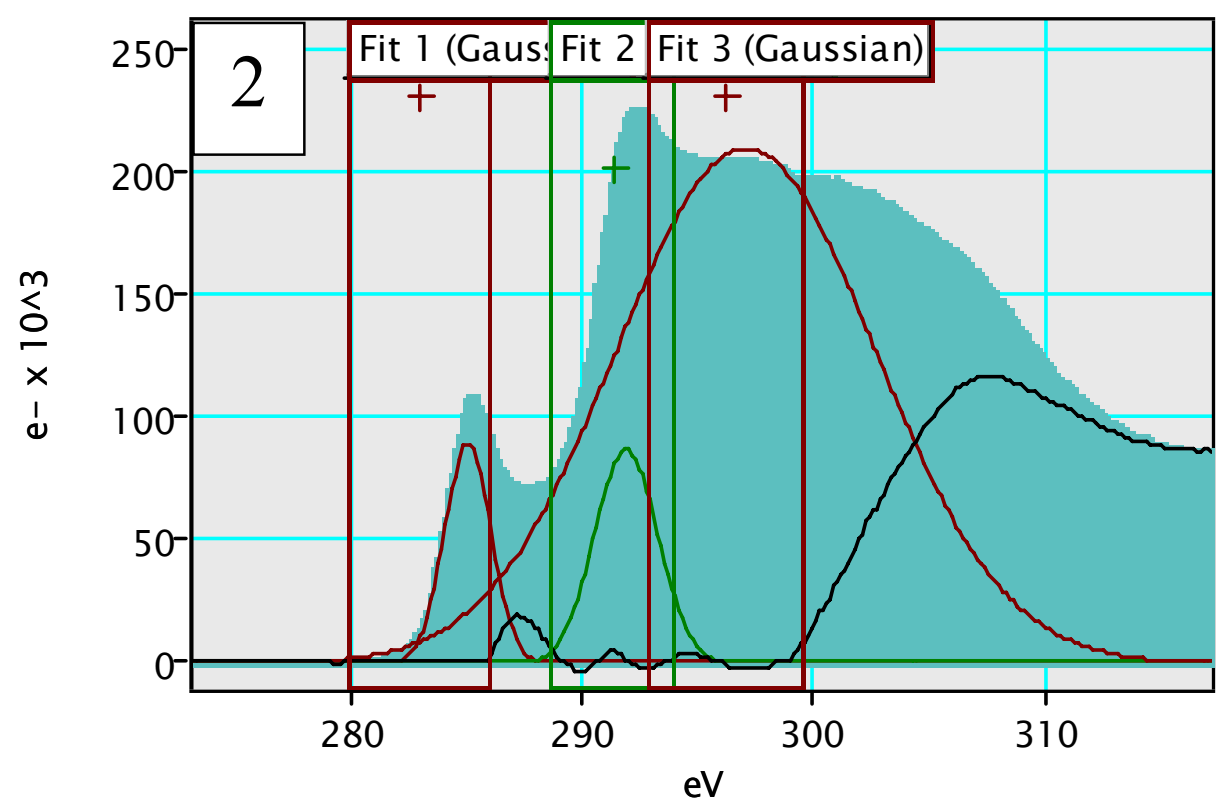

FIG. 2. A carbon $\mathrm{K}$ ionization edge of a disc heat treated at $1600^{\circ} \mathrm{C}$ fitted with three Gaussians. Note the residual peak at ca. $287 \mathrm{eV}$. 